\title{
Abstract: Deep Learning Algorithms Out-perform Veterinary Pathologists in Detecting the Mitotically Most Active Tumor Region
}

\author{
Marc Aubreville ${ }^{1,2}$, Christof A. Bertram ${ }^{3}$, Christian Marzahl ${ }^{2}$, \\ Corinne Gurtner ${ }^{4}$, Martina Dettwiler ${ }^{4}$, Anja Schmidt ${ }^{5}$, \\ Florian Bartenschlager ${ }^{3}$, Sophie Merz ${ }^{3}$, Marco Fragoso ${ }^{3}$, Olivia Kershaw ${ }^{3}$, \\ Robert Klopfleisch ${ }^{3}$, Andreas Maier ${ }^{2}$ \\ ${ }^{1}$ Technische Hochschule Ingolstadt, Germany \\ ${ }^{2}$ Pattern Recognition Lab, Friedrich-Alexander-Universität Erlangen-Nürnberg, \\ Germany \\ ${ }^{3}$ Institute of Veterinary Pathology, Freie Universität Berlin, Germany \\ ${ }^{4}$ Department of Infectious Diseases and Pathobiology, Vetsuisse Faculty, University of \\ Bern, Bern, Switzerland \\ ${ }^{5}$ Vet Med Labor GmbH - Division of IDEXX Laboratories, Ludwigsburg, Germany \\ marc.aubreville@thi.de
}

Manual count of mitotic figures, which is determined in the tumor region with the highest mitotic activity, is a key parameter of most tumor grading schemes. The mitotic count has a known high inter-rater disagreement and is strongly dependent on the area selection due to uneven mitotic figure distribution. In our work[1], we assessed the question, how significantly the area selection could impact the mitotic count. On a data set of 32 cases of H\&E-stained canine cutaneous mast cell tumor, fully annotated for mitotic figures, we asked eight veterinary pathologists to select a field of interest for the mitotic count, and retrieved the mitotic count for that area from the data set. Additionally, we evaluated three deep learning-based methods for the assessment of highest mitotic density. We found that the predictions by all models were, on average, better than those of the experts. Further, we found considerable differences in position selection between pathologists, which could partially explain the high variance that has been reported for the manual mitotic count. To achieve better inter-rater agreement, we propose to use a computer-based area selection for support of the pathologist in the manual mitotic count.

\section{References}

1. Aubreville M, Bertram CA, Marzahl C, et al. Deep learning algorithms out-perform veterinary pathologists in detecting the mitotically most active tumor region. Sci Rep. 2020;10(1):16447. 